\title{
Bioengineered pseudovirus nanoparticles displaying the HA1 antigens of influenza viruses for enhanced immunogenicity
}

\author{
Ming Xia ${ }^{1, \S}$, Md Rejaul Hoq ${ }^{2, \S}$, Pengwei Huang ${ }^{1, \S}$, Wen Jiang ${ }^{2}$, Xi Jiang ${ }^{1,3}$, and Ming $\operatorname{Tan}^{1,3}(\bowtie)$ \\ ${ }^{1}$ Division of Infectious Diseases, Cincinnati Children's Hospital Medical Center, Cincinnati, OH 45229, USA \\ ${ }^{2}$ Department of Biological Sciences, Purdue Cryo-EM Facility, Purdue University, IN 47907, USA \\ ${ }^{3}$ Department of Pediatrics, University of Cincinnati College of Medicine, Cincinnati, OH 45229, USA \\ ${ }^{\S}$ Ming Xia, Md Rejaul Hoq, and Pengwei Huang contributed equally to this work. \\ (C) Tsinghua University Press and Springer-Verlag GmbH Germany, part of Springer Nature 2021 \\ Received: 3 October 2021 / Revised: 19 November 2021 / Accepted: 22 November 2021
}

\begin{abstract}
Even with implementation of current influenza vaccines, influenza still claims up to 500,000 lives worldwide annually, indicating a need for a better vaccine strategy. We have developed a technology to generate unique $S_{60}-\mathrm{HA} 1$ pseudovirus nanoparticles (PVNPs) that display the receptor-binding HA1 domains of influenza viruses. Each self-assembled $\mathrm{S}_{60}-\mathrm{HA} 1 \mathrm{PVNP}$ consists of a $T$ $=1$ icosahedral $\mathrm{S}_{60}$ nanoparticle that resembles the inner shell of norovirus capsid and 60 surface-displayed HA1 antigens that are excellent vaccine targets. Soluble $S_{60}-\mathrm{HA} 1$ PVNPs presenting HA1 antigens of H7N9 influenza virus subtypes have been produced efficiently in large amount. Their three-dimensional (3D) structures have been solved by cryogenic electron microscopy. The PVNP-displayed HA1 antigens react with HA-specific antibody, and retain authentic sialic acid binding specificity and hemagglutinate human erythrocytes. The PVNPs are highly immunogenic, eliciting high titers of HA1-specific antibodies in mice and the mouse sera strongly inhibited hemagglutinations of homologous and heterologous influenza virus $\mathrm{HA}$ proteins. Therefore, the $\mathrm{S}_{60}$-HA1 PVNPs may provide useful reagents to study influenza viruses and offer a potential new vaccine tactic to fight the deadly influenza disease.
\end{abstract}

\section{KEYWORDS}

$\mathrm{S}_{60}$ nanoparticle, pseudovirus nanoparticle, influenza virus, influenza vaccine, hemagglutinin, norovirus

\section{Introduction}

Influenza viruses (IVs), members of the family Orthomyxoviridae, are single-stranded, negative-sense RNA viruses causing deadly, epidemic influenza (flu) disease in both humans and animals. WHO and other organizations estimated that seasonal flu leads to 250,000-500,000 deaths per annum globally despite of applications of the current flu vaccines [1-3]. Thus, flu continues to be a significant global health burden, and new flu control and prevention strategies with improved efficacy are urgently needed. Structurally, each virus is covered by a lipid envelope, two surface proteins, known as hemagglutinin (HA) and neuraminidase (NA), respectively, protrude outward from the envelope, forming the viral spikes. They are numbered distinctly to name various influenza A virus (IAV) subtypes through different HA-NA combinations. The HA and NA play key roles in IV replication cycles, and thus are excellent targets of vaccines and antiviral drugs.

Vaccines remain the most effective approach to control and prevent flu. However, US Centers for Disease Control and Prevention (CDC) records indicate that the effectiveness of conventional flu vaccines based on egg-grown, inactivated IVs was low, ranging from $10 \%$ to $60 \%$ [4], explaining the continual flu prevalence with significant mortality and morbidity. Most of the vaccines are produced by growing the corresponding IV strains in fertilized chicken eggs, which often leads to adaptive mutations and thus altered antigenicity of the vaccine IVs after many cycles of propagations in eggs. Such mutations have been shown to be an important factor contributing to the low effectiveness of egg-based flu vaccines [5-8].

For example, the egg-grown contemporary $\mathrm{H} 3 \mathrm{~N} 2$ vaccine strains used since 2016 were found antigenically mismatched circulating $\mathrm{H} 3 \mathrm{~N} 2$ strains $[9,10]$, explaining the low efficacy of H3N2 vaccine during the winter seasons of 2016/2017 (33\%) and $2017 / 2018$ (22\%) [4, 11]. Accordingly, a study to compare human H3N2 antibody responses elicited by egg-based (Fluzone ${ }^{\circledR}$ ), cellbased (Flucelvax ${ }^{\odot}$ ), and recombinant HA-based (Flublok ${ }^{\otimes}$ ) flu vaccines during the $2017 / 2018$ winter season showed that the recombinant HA-based flu vaccines exhibited significantly higher neutralizing antibody titers than those induced by the egg- and cellbased flu vaccines [8]. Therefore, the recombinant HA-based flu vaccine with original HA sequences represents an approach for higher protective efficacy than the egg/cell-grown vaccines with adaptative mutations.

The immunodominant HA1 domains constitute the heads of IV HA trimers, interacting with host sialic acid receptors to initiate IV infection and thus are an ideal vaccine target. The commercial use of the Flublok ${ }^{\oplus}$ vaccine has demonstrated the feasibility and usefulness of the recombinant HA-based flu vaccine approach. However, the relatively smaller size and low valence of a 
recombinant HA trimer may be less immunogenic compared with the polyvalent HA antigens on a virus particle. This problem could be solved through displays of the HA antigens by a polyvalent protein nanoparticle platform, such as a 24 -valent nanoparticle [12-14] formed by ferritins that are ubiquitous iron storage proteins, or the 60-valent I53-50 nanoparticle [15] that is one of several computationally designed complexes [16]. Alternatively, the HA antigens were displayed by lipid nanoparticles [17-19]. The results from these studies showed that the polyvalent platforms enhanced the immune responses of the displayed antigens. These nanoparticle-based vaccines are currently under development.

Recently, we have developed a $T=1$ icosahedral $\mathrm{S}_{60}$ nanoparticle that is self-assembled by 60 norovirus (NoV) capsid shell (S) domains with their C-termini exposed on the surface in triangle patterns [20]. This 60 -valent nanoparticle resembles the inner shell of NoV capsid and has been shown to be a useful platform to display VP8* antigens, the receptor-binding domains of rotaviruses, for improved immunogenicity as a rotavirus vaccine candidate $[20,21]$. In this study, the receptor-binding HA1 domains of IV HAs were fused to the NoV S domains to generate $\mathrm{S}_{60}$-HAl pseudovirus nanoparticles (PVNPs) with 60 exposed HA1 antigens arranged into 20 HA1-trimers, resembling those on IV virions. We have established methods to generate the self-assembled $\mathrm{S}_{60}-\mathrm{HA} 1$ PVNPs displaying $\mathrm{HA1}$ antigens of H7N9 IAV subtype. The PVNP-presented HAls are recognized by HAspecific antibody, retain authentic receptor binding function, and agglutinate human red blood cells (RBCs). The PVNPs are highly immunogenic, eliciting high titers of HA1-specific antibody that inhibited hemagglutinations of HA proteins, supporting the notions that the $\mathrm{S}_{60}-\mathrm{HA} 1$ PVNPs may be used as reagents for IV study and that they may serve as a flu vaccine candidate.

\section{Materials and methods}

\subsection{Plasmids for S-HA1 fusion protein expressions}

DNA sequences encoding the HA1 antigen of an A/H7N9 strain (GenBank AC\#: AUN86892.1, A/Jiangsu/TM261/2017/H7N9, amino acid C60 to N285, 226 aa), were codon-optimized to Escherichia Coli using the codon adaptation tool at http://www.jcat.de and synthesized by GenScript (Piscataway, NJ). The DNA fragment was cloned into the previously made pET-24b (Novagen)-based plasmid that was generated for production of the $\mathrm{S}_{\mathrm{R} 69 \mathrm{~A}}-\mathrm{VP}^{\star}$ fusion protein [20] by replacing the VP8*-encoding sequences using the HA1-encoding DNA fragment. R69A refers to the mutation in the NoV S domain to remove the exposed protease site [20]. A linker (HHHH) was added between the $S$ and HA1 domains and a His tag was fused to the C-terminus of HA1 (Fig. 1(a)). A further plasmid without the His-tag encoding sequences was also made for tag-free S-HA1 protein production. As a control for comparisons, a further plasmid for expression of glutathione S-transferase (GST)-HA1 fusion protein were made by cloning the HA1-encoding DNA fragment into the pGEX-4T-1 vector (GE Healthcare Life Sciences) [22].

\subsection{Tagged protein production}

Recombinant proteins were expressed using the E. coli (BL21, Arctic) system induced by $0.4 \mathrm{mM}$ isopropyl- $\beta$-Dthiogalactopyranoside (IPTG) at $13{ }^{\circ} \mathrm{C}$ overnight as described previously $[20,23,24]$. Soluble His-tagged proteins were purified with His-tag binding cobalt resins (Thermo Fisher Scientific, Waltham, MA, USA) according to the instructions of the manufacturers, eluted with $150 \mathrm{mM}$ imidazole (Sigma-Aldrich, St. Louis MO, USA) in $50 \mathrm{mM}$ phosphate buffered saline (PBS, $\mathrm{pH}$

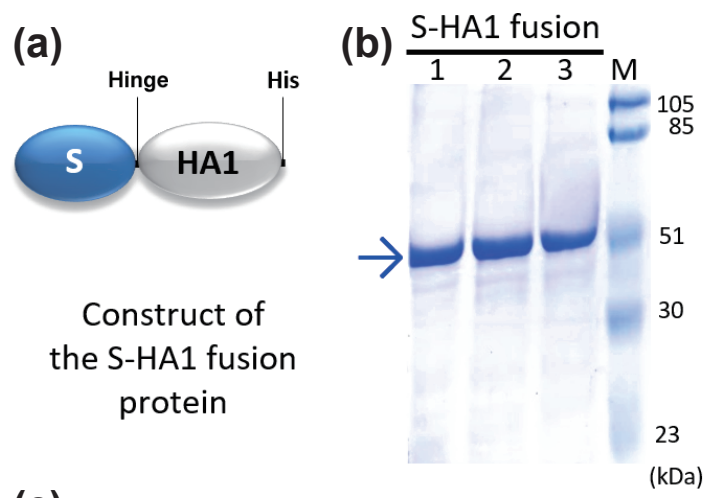

(c)
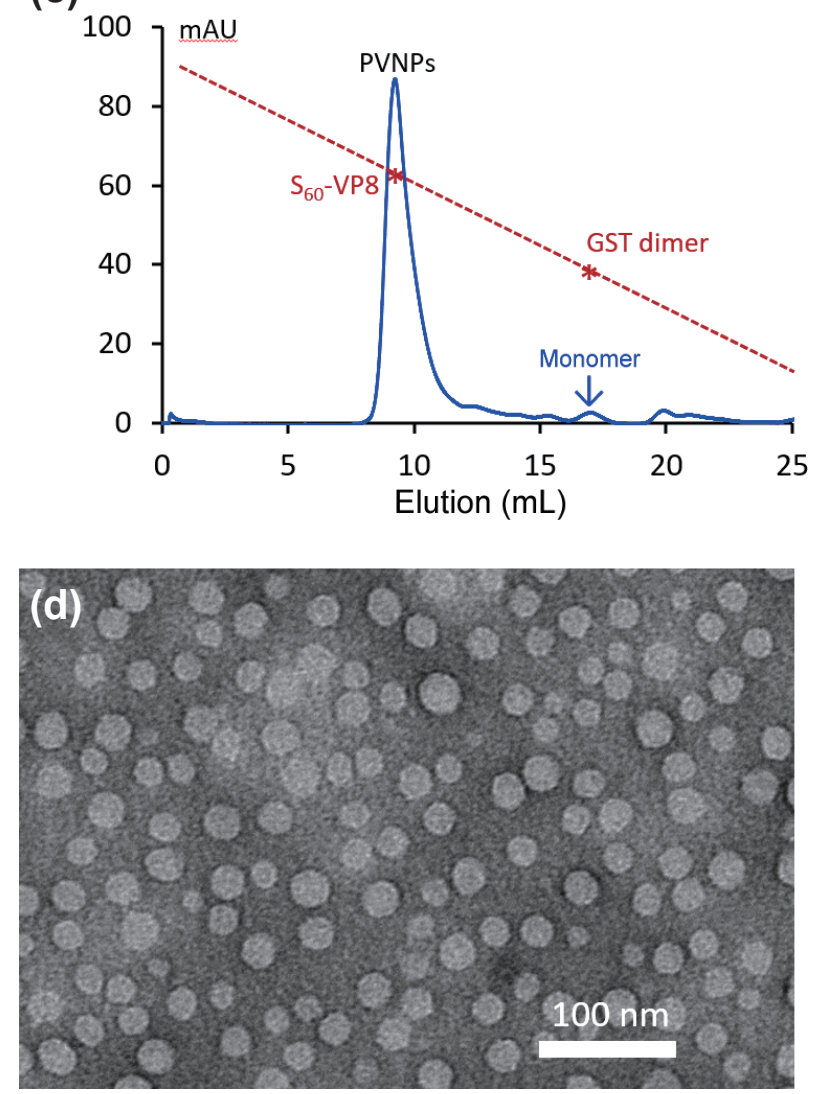

Figure 1 Production and characterization of His-tagged S-HA1 PVNPs. (a) Schematic construct of the His-tagged S-HA1 proteins. S, modified norovirus S domain; hinge, the hinge region of norovirus VP1; HA1, the HA1 antigen of H7 subtype; His, His tag. (b) SDS-PAGE of the purified S-HAl protein (arrow) at $52 \mathrm{kDa}$. Lanes $1 / 2 / 3$, purified proteins of three preparations; $\mathrm{M}$, protein standards with indicated molecular sizes. (c) An elution curve of gel-filtration chromatography of the purified S-HAl protein through a size exclusion column. The major PVNP elution peak and a minimal peak of S-HA1 protein monomers are indicated. The two elution peaks were calibrated using the $\mathrm{S}_{60^{-}}$ VP8 $^{*}$ nanoparticles $(\sim 3.4 \mathrm{mDa})$ [20] and GST dimers $(52 \mathrm{kDa})$ with their elution positions shown by star symbols on a red dashed line. $Y$-axis shows UV $\left(\mathrm{A}_{280}\right)$ absorbances (mAU), while $X$-axis indicates elusion volume (ml). (d) A representative micrograph of transmission electronic microscopy (TEM) showing the S-HA1 PVNPs.

7.2). Soluble GST-tagged proteins were purified using the GSTbinding resin (Glutathione Sepharose 4B GST-tagged protein purification resin, GE Healthcare Life Sciences) as described previously [24].

\subsection{Tag-free protein production}

The tag-free S-HA1 protein was expressed in E. coli as described above. For purification, clarified bacterial lysis was incubated with ammonium sulfate $\left[\left(\mathrm{NH}_{4}\right)_{2} \mathrm{SO}_{4}\right]$ at $1.2 \mathrm{M}$ end concentration for 30 min to selectively precipitate the S-HA1 proteins as reported 
previously [25]. Briefly, after centrifugation with an Avanti J26XP centrifuge (Beckman Coulter) using a JA-17 rotor at 10,000 rpm for $20 \mathrm{~min}$, the protein pellet was collected, washed twice using 1.2 $\mathrm{M}\left(\mathrm{NH}_{4}\right)_{2} \mathrm{SO}_{4}$ solution (in PBS), and then solved in $20 \mathrm{mM}$ tris buffer ( $\mathrm{pH}$ 7.5). The protein was analyzed and further purified by gel-filtration, ion exchange chromatography, and cesium chloride $(\mathrm{CsCl})$ density gradient centrifugation (see below).

\subsection{Sodium dodecyl sulfate polyacrylamide gel electrophoresis (SDS-PAGE) and protein quantitation}

Purified proteins were analyzed by SDS-PAGE using 10\% separating gels and were quantitated by SDS-PAGE using serially diluted bovine serum albumin (BSA, Bio-Rad) with known concentrations as standards on same gels [26].

\subsection{Gel filtration chromatography}

This was performed as described previously $[23,24,27]$ using an AKTA Fast Performance Liquid Chromatography system (FPLC, AKTA Pure 25L, GE Healthcare Life Sciences) using a size exclusion column (Superdex 200, 10/300 GL, $25 \mathrm{~mL}$ bed volume, GE Healthcare Life Sciences) to analyze the sizes of the S-HA1 proteins as evidence of PVNP formation. The column was calibrated using gel filtration calibration kits (GE Healthcare Life Sciences), while the elution peaks of the PVNPs and S-HA1 monomers were determined using the previously made $\mathrm{S}_{60}$-VP8 ${ }^{*}$ nanoparticles ( $3.4 \mathrm{mDa}$ ) [20] and GST-dimer (52 kDa). Relative protein concentrations in the effluent were reported as $A_{280}$ absorbance.

\subsection{Anion exchange chromatography}

This was carried out using the same AKTA FPLC system (see above) using a HiPrep Q HP 16/10 column (20 mL bed volume, GE Healthcare Life Sciences) to analyze and purify the tag-free SHA1 protein. A previous established procedure [25] was modified and applied here. After the column was equilibrated using 7 column volumes (CVs) of $20 \mathrm{mM}$ Tris buffer ( $\mathrm{pH}$ 8.0, referred as buffer A), protein samples were loaded. The column was washed using $7 \mathrm{CVs}$ of buffer $\mathrm{A}$ to remove the unbound proteins, followed by elution of the bound proteins using 7 CVs of buffer $B$ ( $1 \mathrm{M} \mathrm{NaCl}$ in buffer A) via a linear gradient elution manner $(0 \%-100 \%$ B). The column was then stripped by 7 CVs of buffer B (100\% B), followed by an equilibration using 7 CVs of buffer A. Relative protein concentrations in the effluent were reported as $\mathrm{A}_{280}$ absorbance, while the elution positions of the proteins were shown as percentages of buffer $B$.

\section{7 $\mathrm{CsCl}$ density gradient ultracentrifugation}

This was performed as described previously [20]. Briefly, $0.5 \mathrm{~mL}$ of $\left(\mathrm{NH}_{4}\right)_{2} \mathrm{SO}_{4}$ precipitated S-HAl protein (see above) was mixed with $10 \mathrm{~mL}$ of $\mathrm{CsCl}$ solution with a density of $1.3630 \mathrm{~g} / \mathrm{mL}$ and packed in a $12-\mathrm{mL}$ centrifuge tube. After centrifugation at 288, $000 \times g$ for $45 \mathrm{~h}$ in the Optima L-90K ultracentrifuge (Beckman Coulter), the gradient was fractionated by bottom puncture into 21 fractions $(\sim 0.5 \mathrm{~mL}$ each). $\mathrm{CsCl}$ densities of fractions were determined according to the refractive index.

\subsection{Transmission electron microscopy (TEM)}

TEM was used to inspect the morphology of the PVNPs. After staining with $1 \%$ ammonium molybdate, purified S-HA1 proteins were observed under an EM10 C2 microscope (Zeiss, Germany) at $80 \mathrm{kV}$ at magnifications between $15,000 \times$ and $30,000 \times$ as described elsewhere [20].

\subsection{Immunization of mice}

Specific pathogen free (SPF) BALB/c mice at $\sim 6$ weeks of age were purchased from the Jackson Laboratory (Bar harbor, ME, USA) and maintained under SPF conditions at the Division of Veterinary Services of Cincinnati Children's Hospital Medical Center (CCHMC). Mice were randomly divided into four groups with 6-8 mice each $(n=6-8)$ and each group was administered with one of following immunogens: (1) $\mathrm{S}_{60}$-HA1 PVNPs $\left(\mathrm{S}_{60^{-}}\right.$ HA1); (2) GST-HA1 fusion protein (GST-HA1); (3) $S_{60}$ nanoparticle without $\mathrm{HA1}$ antigens as platform control $\left(\mathrm{S}_{60}\right)$; and (4) PBS as diluent control (PBS). Immunogens were administered with Alum adjuvant (Imject Alum, Thermo Fisher Scientific, USA) at $25 \mu \mathrm{L} /$ dose $(20 \mu \mathrm{g} /$ mouse/dose $)$. Endotoxin contaminations of the recombinant protein immunogens were removed using Endotoxin Removal Spin Columns (Thermo Scientific ${ }^{\text {TM }}$ Pierce, USA). After this treatment, all immunogens for mouse immunization contained less than 1.5 endotoxin units. Mice were immunized intramuscularly (i.m.) in the thigh muscle three times with injection volume of $50 \mu \mathrm{L}$ at 2-week intervals. Bloods were collected 2 weeks after the final immunization via heart puncture and sera were processed from bloods via an established protocol [26].

\subsection{Enzyme immunoassays (EIAs)}

EIAs were used to determine HA1 specific antibody titers as described elsewhere $[28,29]$. Briefly, commercial recombinant $\mathrm{H} 7$ HA protein (Sino Biological) was coated on 96-well plates at 1 $\mu \mathrm{g} / \mathrm{mL}$. After blocked with $5 \%(\mathrm{w} / \mathrm{v})$ skim milk, plates were incubated with mouse sera at serial dilutions. The bound antibodies were measured by goat-anti-mouse IgG-horse radish peroxidase (HRP) conjugate (1:5,000, MP Biomedicals). IgG titers were defined as maximum dilutions of sera with positive signals. A positive signal was determined by a mean of negative values $+3 \times$ standard deviation. EIAs were also used to measure S-HA1 PVNPs in the fractions of $\mathrm{CsCl}$ density gradient. In this case, the fractions were diluted in 800 folds in PBS and coated on microtiter plates. The PVNPs were detected using our in-house made antibody against NoV-like particle (VLP) [30].

\subsection{Cryogenic electron-microscopy (cryoEM)}

CryoEM was carried out to reconstruct the three-dimensional (3D) structures of the S-HA1 PVNPs as described in our previous studies [26, 31,32]. Briefly, $3 \mu \mathrm{L}$ of purified PVNPs were dropped onto graphene oxide coated lacey grids (Tedpella \#01896), then blotted for $4 \mathrm{~s}$ before flashed frozen into ethane using Cp3 cryoplunder (Gatan, Inc., Pleasanton CA, USA) at 90\% humidity. Lowelectron-dose images $\left(\sim 20\right.$ e/ $\left./ \AA^{2}\right)$ were recorded on Talos F200C cryo-TEM, recorded by Ceta $16 \mathrm{M}$ camera at a nominal magnification $(57,000 \times)$ with a calibrated pixel size of $2.59 \AA$. The collected micrographs were imported into cryoSPARC/v3 for further processing. A total of $>14,000$ particles were selected with Gautomatch v0.53 (http://www.mrc-lmb.cam.ac.uk/kzhang/). Multiple homogeneous class averages were separated using twodimensional (2D) classification in CryoSPARC 3.1 [33]. The Histagged particles were further divided to two classes based on their size difference, among which 6,200 separated particles for the larger, and 5,284 particles for the smaller PVNPs were subjected to ab-initio 3D reconstruction for initial model generation and final homogeneous refinement for further analysis. Similarly, 1,064 separated tag-free PVNPs particles were selected for initial model generation, followed by homogeneous refinement. CryoEM map fitting was conducted using UCSF Chimera software (http://www.rbvi.ucsf.edu/chimera; version 1.15) using in the crystal structures of the inner shell of the 60-valent feline calicivirus (FCV) VLP (PDB code: 4PB6) [34] and the HA1 heads of $\mathrm{H} 7 \mathrm{HA}$ trimer (PDB code: 4LN3) $[35,36]$. The structure of the $\mathrm{S}_{60}$ nanoparticle was modeled using the crystal structure of the 60 - 
valent FCV inner shell (PDB code: 4PB6) using the same UCSF Chimera software.

\subsection{EIA-based glycan binding assay}

This was used to measure interactions between the S-HA1 PVNPs and sialoglycans. Sialoglycans of (1) Neu5Aca2-8Neu5Aca28Neu5Aca-sp-PAA-biotin, (2) Neu5Ac $\beta 2,6(\mathrm{Gal} \beta 1,3)$ GalNAcaPAA-biotin, (3) $\alpha$-Neu5Ac2,3ßGall,4Glc(3'-sialyllactose)-PAAbiotin, (4) sialyl Le $\mathrm{a}^{\mathrm{a}}$-PAA-biotin, and (5) sialylLe $\mathrm{e}^{\mathrm{x}}$-PAA-biotin were from GlycoTech (Rockville, MD), while glycans of Neu5Aca2, 3Gal 31 ,3(Fuca1,4)GlcNAc $\beta$-SpNH-PAA-biotin and Neu5Aca2, 3Gal 1 1,4(Fuca1,3)GlcNAc $\beta$-SpNH-PAA-biotin were from the Consortium for Functional Glycomics (http://www.functionalglycomics.org/). All glycans were conjugated with polyacrylic acid (PAA) and labeled with biotin. Both PVNPs and the $S_{60}$ nanoparticle control at $10 \mu \mathrm{g} / \mathrm{mL}$ were coated on microtiter plates (Thermo Scientific) at $4{ }^{\circ} \mathrm{C}$ overnight. After blocking with $5 \%(\mathrm{w} / \mathrm{v})$ nonfat milk, the plates were incubated with the glycans at $2 \mu \mathrm{g} / \mathrm{mL}$ for $60 \mathrm{~min}$. The bound glycans were detected by streptavidin-HRP conjugates (Jackson ImmunoResearch Laboratories) at 1:5,000 dilution.

\subsection{EIA-based assays of antigenic reactivity}

This was performed to test the antigenic reactivity of the S-HA1 PVNPs with HA specific antibody as described elsewhere [22]. Briefly, native and boiled S-HA1 PVNPs were serially diluted and coated on microtiter plates (Thermo Scientific ${ }^{\mathrm{TM}}$, USA) at $4{ }^{\circ} \mathrm{C}$ overnight. After blocking, commercial H7 HA specific antibody (Sino Biological) at 1:1,000 or appropriate dilutions was added to the coated wells. The bound antibody was detected by corresponding HRP-conjugated secondary antibody (Thermo Scientific $^{\mathrm{TM}}, \mathrm{USA}$ ) at 1:2,000 dilution.

\subsection{Hemagglutination and hemagglutination inhibition (HI) assays}

The assays were used measure hemagglutination titers of recombinant HAs and S-HA1 PVNPs using human RBCs that were provided by the Translational Core Laboratory at CCHMC, as described elsewhere [22]. Briefly, $500 \mu \mathrm{L}$ of $0.5 \%$ RBCs in 10 mM PBS were mixed with serially diluted HAs or PVNPs in 96well V-bottom plates and incubated at $4{ }^{\circ} \mathrm{C}$ for $60-100 \mathrm{~min}$, using the $S_{60}$ nanoparticles and PBS as negative controls. Hemagglutination titers were defined as the highest dilutions of the HAs or PVNPs that caused hemagglutination. For HI assays, HAs (Sino biological) or PVNPs at $4 \times$ hemagglutination titers were incubated with serially diluted serum antibody for $60 \mathrm{~min}$ before they were mixed with RBCs for hemagglutination assays. The $\mathrm{HI}$ titers were defined as the maximum serum dilutions that prevented hemagglutination.

\subsection{Ethics statement}

All animal studies were completed in compliance with the recommendations in the Guide for the Care and Use of Laboratory Animals (23a) of the National Institute of Health $(\mathrm{NIH})$. The protocols were approved by the Institutional Animal Care and Use Committee (IACUC) of the Cincinnati Children's Hospital Research Foundation (Animal Welfare Assurance No. A3108-01).

\subsection{Statistical analysis}

Statistical differences between two data groups were calculated by GraphPad Prism 9.0 (GraphPad Software, Inc.) using unpaired Student's $t$-test. Differences were considered non-significant when $P$-values $>0.05$ (marked as ns), significant when $P$-values $<0.05$ (marked as ${ }^{*}$ ), highly significant when $P$-values $<0.01$ (marked as ${ }^{* *}$ ), and extremely significant when $P$-values $<0.001$ (marked as $\left.{ }^{* *}\right)$, respectively.

\section{Results}

\subsection{Production of His-tagged S-HA1 PVNPs}

S-HA1 fusion protein containing the HA1 antigens of an H7N9 IV (Fig. 1(a)) was produced via the E. coli system. The Cterminally His-tagged, soluble S-HA1 protein was purified at yields of $\sim 20 \mathrm{mg} / \mathrm{L}$ bacterial culture using His-tag binding cobalt resin. SDS-PAGE showed the purified protein at an expected size of $\sim 52 \mathrm{kDa}$ (Fig. 1(b)). Gel-filtration chromatography of the protein revealed a single major elution peak with high molecular size like the previously made $\mathrm{S}_{60}-\mathrm{VP} 8^{*}$ nanoparticles [20] (Fig. 1(c)), while the elution peak corresponding to the S-HA1 protein monomers was minimal, indicating that vast majority of the proteins self-assembled into PVNPs or large complexes. TEM inspection of the major gel-filtration elution peak demonstrated the self-formation of the S-HA1 H7 PVNPs (Fig. 1(d)). Major PVNP population had sizes between 16 and $20 \mathrm{~nm}$ with some variations.

\subsection{Generation of tag-free S-HA1 H7 PVNPs}

To assess potential impact of the His-tag on PVNP formation and explore alternative production and purification approaches, tagfree S-HA1 protein (Fig. 2(a)) was also produced and purified by selective precipitation using $\left(\mathrm{NH}_{4}\right)_{2} \mathrm{SO}_{4}$ [25] at concentrations of 0.7 and $1.2 \mathrm{M}$. This resulted in high protein yields at $30-40 \mathrm{mg} / \mathrm{L}$ bacterial culture (Fig. 2(b)). Gel-filtration of the $\left(\mathrm{NH}_{4}\right)_{2} \mathrm{SO}_{4^{-}}$ purified, resolved protein revealed a similar single major elution peak of PVNPs (Fig. 2(c)), confirming that the fusion protein selfassembled into PVNPs. The protein was also analyzed by an anion exchange chromatography (Fig. 2(e)), in which vast majority of the target protein was eluted in the major peak corresponding to $31.7 \%$ buffer $\mathrm{B}$, equivalent to an ionic strength of $317 \mathrm{mM} \mathrm{NaCl}$ and $20 \mathrm{mM}$ Tris. SDS-PAGE of the major elution peaks of the gelfiltration and ion exchange revealed the target proteins at high purity (Fig.2(d)), supporting that the two chromatography approaches may be used to further purify the $\left(\mathrm{NH}_{4}\right)_{2} \mathrm{SO}_{4}{ }^{-}$ precipitated, tag-free S-HA1 proteins.

The $\left(\mathrm{NH}_{4}\right)_{2} \mathrm{SO}_{4}$-purified protein was analyzed further by $\mathrm{CsCl}$ density gradient centrifugation, resulting in a visible protein band at a density of $\sim 1.311 \mathrm{mg} / \mathrm{cm}^{3}$ (Fig. 2(f)). The gradient was fractionated into 21 portions, followed by detection of the PVNPs in the factions by EIA assays using the $\mathrm{S}$ domain-specific antibody, confirming the protein band as the S-HA1 protein (Fig. $2(\mathrm{~g})$ ). Finally, the protein band was examined by TEM, revealing typical PVNPs (Fig. 2(h)) with better homogeneity than the His-tagged ones (comparing Fig. 2(h) with Fig. 1(d)). These data indicated that, while the His-tags did not substantially affect the PVNP formation, tag-free PVNPs showed better production yields and improved PVNP homogeneity.

\subsection{Structures of the His-tagged S-HA1 PVNPs}

The 3D structures of the S-HA1 PVNPs were reconstructed using cryoEM approach. By focusing on the major populations, we noted and reconstructed two types of the His-tagged PVNPs that have distinct diameters of $\sim 21$ and $\sim 16 \mathrm{~nm}$, respectively. Both PVNPs were reconstructed at 12- $\AA$ resolution (Figs. 3 and 4) and they are in $T=1$ icosahedral symmetry, consisting of 60 S-HA1 proteins, thus are named $\mathrm{S}_{60}-\mathrm{HA} 1$ PVNPs. Fitting of the crystal structure of the $S_{60}$-nanoparticle (PDB ID: 4PB6) $[20,34]$ into the cryoEM density map of the 21-nm PVNP inner shell region 


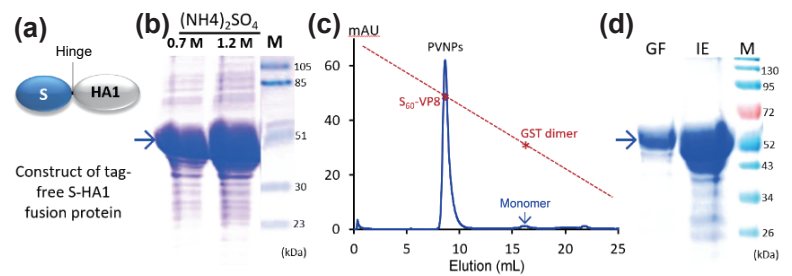

(e)

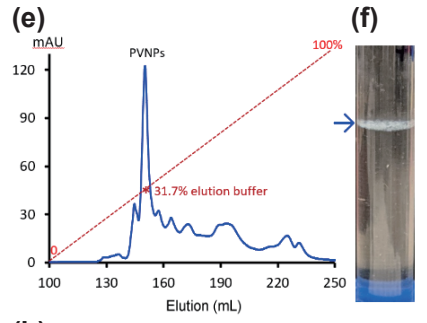

(h)

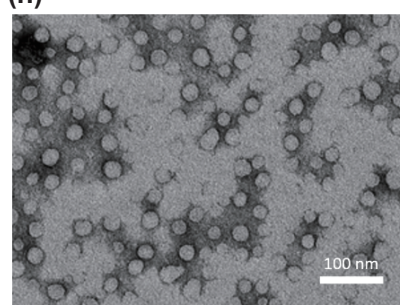

Figure 2 Generation and characterization of tag-free S-HA1 PVNPs. (a) Schematic construct of the tag-free S-HA1 protein. S, modified norovirus S domain; hinge, the hinge region of norovirus VP1; HA1, the HA1 antigen. (b) SDS-PAGE of $\left(\mathrm{NH}_{4}\right)_{2} \mathrm{SO}_{4}(0.7$ and $1.2 \mathrm{M})$ precipitated tag-free S-HA1 proteins (arrow). M, protein standards with indicated molecular sizes. (c) A gel-filtration elution curve of the $\left(\mathrm{NH}_{4}\right)_{2} \mathrm{SO}_{4}$-purified S-HA1 protein through a size exclusion column. The major PVNP elution peak and the minimal S-HA1 monomers peak (arrow) are shown. The elution peaks were calibrated using the $\mathrm{S}_{60}-\mathrm{VP}^{*}$ nanoparticles $(\sim 3.4 \mathrm{mDa})$ [20] and GST dimers $(52 \mathrm{kDa})$ with their elution positions shown by star symbols on a red dashed line. $Y$-axis shows $U V\left(\mathrm{~A}_{280}\right)$ absorbances (mAU), while $X$-axis indicates elusion volume $(\mathrm{mL})$. (d) SDSPAGE of the major peaks of the gel-filtration (GF) and ion exchange (IE) chromatography. M, protein standards. (e) An anion exchange elution curve of the tag-free S-HAl protein. $X$ - and $Y$-axes are explained as in (c). The red dashed line shows the linear gradient increase of the elution buffer $B$ $(0 \%-100 \%)$ with a star symbol showing the percentage of the elution buffer B corresponding to the elution of the PVNPs. The major PVNP elution peak is indicated. (f) and (g) $\mathrm{CsCl}$ density gradient centrifugation of the tag-free S-HA1 protein. (f) A photo of the $\mathrm{CsCl}$ density gradient after centrifugation with a visible protein band (arrow). (g) The gradient was fractionated into 21 portions showing their relative locations compared with (f). The relative S-HA1 protein concentrations of the fractions were determined by EIA. $Y$-axis shows the fraction numbers, while $X$-axis indicates signal intensities (OD) with a red dashed line showing the cut-off signal at $\mathrm{OD}=0.1$. (h) A TEM micrograph of the S-HA1 protein of fraction 15 showing the tag-free S-HA1 PVNPs.

indicated that their shapes match well, but the PVNP inner shell appears slightly smaller, particularly in the protruding regions at the five-fold axes (Fig. 3(c)). This confirmed the identity of the $\mathrm{S}_{60^{-}}$ nanoparticle inner shell of the PVNP (Fig. 3, comparing 3(b) with $3(\mathrm{~h})$ ) and revealed that the surface displayed HA1 antigens force the wall of the $\mathrm{S}_{60}$-nanoparticle to go inward structurally, resulting in a compact interior shell. Similar scenario also occurred to the 16$n m$, His-tagged PVNP, in which both the wall of the $S_{60^{-}}$ nanoparticle shell and HA1 antigens go further inward, leading to a more compact PVNP with smaller diameter (Figs. 4(a) $-4(\mathrm{c})$ ).

The above fitting also showed that the $60 \mathrm{HAl}$ antigens are located along the sunken grooves among the protrusions at the five-fold axes of the $\mathrm{S}_{60}$ nanoparticle (Figs. 3(c), 3(g), 4(e)). Noteworthy, the $60 \mathrm{HAl}$ antigens are arranged into 20 trimers on each PVNP, corresponding to the triangle patterns of the exposed C-termini of the NoV S domains on the $\mathrm{S}_{60}$ nanoparticle (Figs. 3(g), 3(h), 4(e), 4(f)). These trimer-like HAls may help to retain their authentic structures and receptor-binding function (see
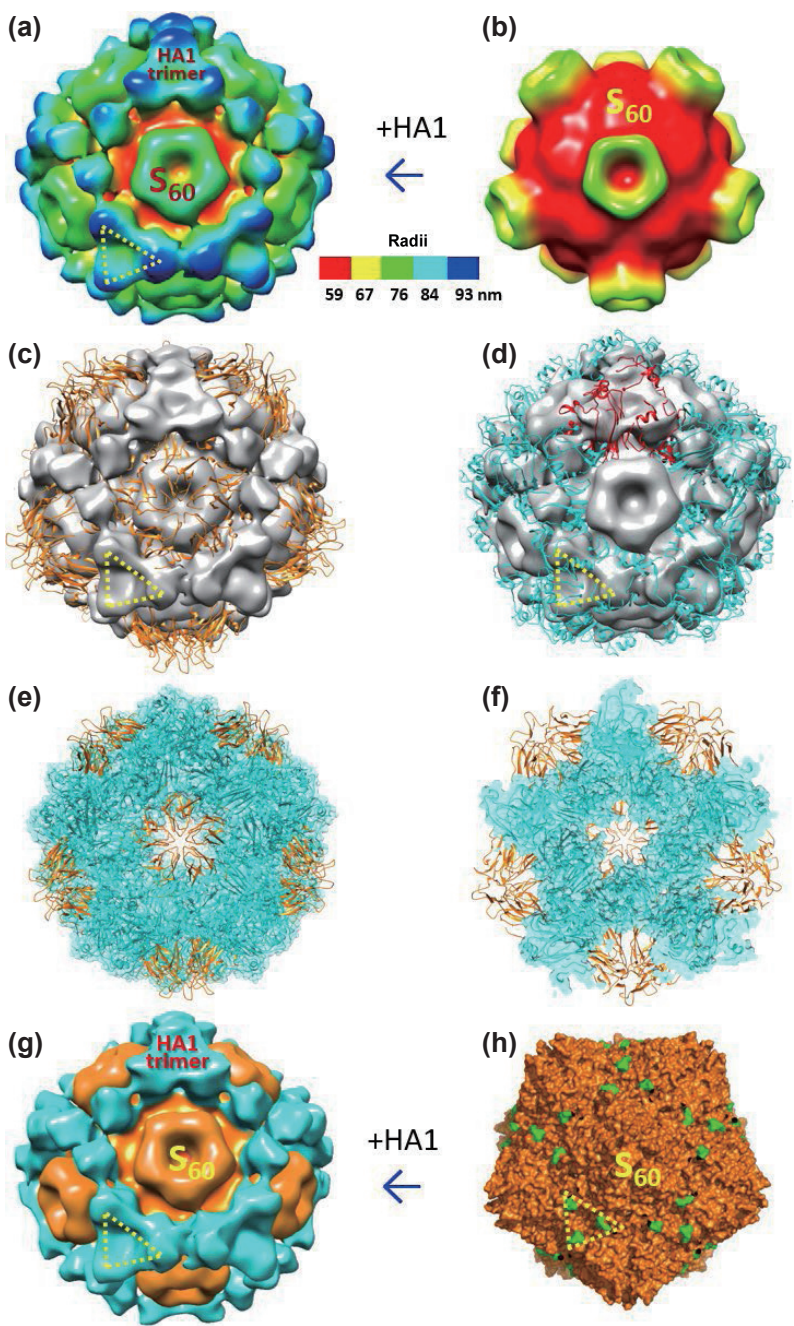

Figure 3 Structures of the His-tagged, 21-nm S60-HA1 PVNPs. (a) and (b) The 3D structures of the 21-nm S $\mathrm{S}_{60}-\mathrm{HA1}$ PVNP (a) and its $\mathrm{S}_{60}$ nanoparticle core (b) reconstructed by cryoEM method, revealing a $T=1$ icosahedral symmetry. The images are shown at five-fold axes. The colored bar in between indicates the radii of the structures. A surface HA1 trimer is indicated by a yellow dashed triangle. (c) Fitting of the crystal structure of the inner shell of the 60 valent feline calicivirus capsid (PDB ID: 4PB6, orange, cartoon representation) into the cryoEM density map of the $S_{60}$ nanoparticle region. (d) Fitting of the crystal structures of HA1 trimers of an H7N9 flu virus (PDB ID: 4LN3, cyan, cartoon representation) into the cryoEM density maps of the surface HA1 trimer regions with one HAl trimer shown in red. (e) and (f) The structural model of the $\mathrm{S}_{60}-\mathrm{HA} 1$ PVNP in PDB format resulted from the above fitting showing a transparent front (e) and a transparent cut (f) views at five-fold axes. The inner $S_{60}$ nanoparticle shell is shown in orange cartoon representation, while the HA1 antigens are shown in transparent surface model with carron representation inside (cyan). (g) Structure of the $\mathrm{S}_{60}-\mathrm{HAl}$ PVNP in two color schemes, showing the $\mathrm{S}_{60}$ nanoparticle core (orange) and the 60 surface-displayed HA1 antigens (cyan), forming $20 \mathrm{HAl}$ trimers. (h) An $\mathrm{S}_{60}$ nanoparticle model (orange) based on the crystal structure of the $T=1$ VLP of feline calicivirus (PDB ID: 4PB6), showing the 60 exposed C-termini (green) of the $\mathrm{S}$ domain in triangle patterns (shown by a yellow dashed triangle). The corresponding HA1 trimers are shown in the $\mathrm{S}_{60}-\mathrm{HA} 1$ images in (a), (c), (d), and (g)

below). When the crystal structures of H7N9 HA1 trimers (PDB ID: 4LN3) [36] were fitted into the cryoEM density maps of the trimer-like HA1 regions, they fitted fine despite some discrepancies on the outside edges (Figs. 3(d) and 4(d)), confirming their structural identities and features. The PVNP structural model in PDB format resulted from the fitting indicated a smaller central lumen than that of the $\mathrm{S}_{60}$-nanoparticle [20] (Figs. 3(e) and 3(f)). Finally, the two $\mathrm{S}_{60}-\mathrm{HA1}$ PVNPs in two color schemes were generated to show the inner shell and the surface trimeric HA1 antigens, respectively (Figs. 3(g) and 4(e)). 

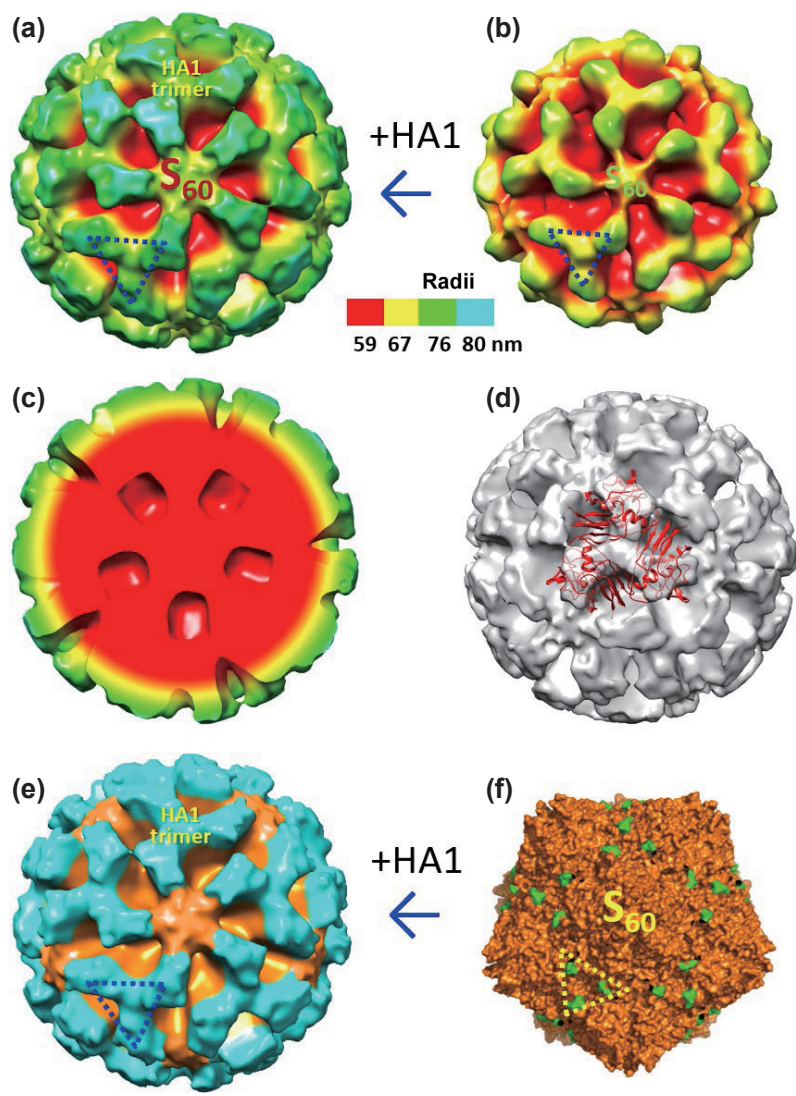

Figure 4 Structures of the His-tagged, 16-nm $\mathrm{S}_{60}-\mathrm{HA1}$ PVNPs. (a) and (b) The 3D structures of the 16-nm $\mathrm{S}_{60}-\mathrm{HA} 1 \mathrm{PVNP}(\mathrm{a})$ and its $\mathrm{S}_{60}$ nanoparticle core region (b) reconstructed by cryoEM method, revealing a $T=1$ icosahedral symmetry. The images are shown at five-fold axes. The colored bar in between indicates the radii of the structures. A surface HAl trimer is indicated by a dashed triangle. (c) The cutting view of a PVNP showing its central structure. (d) Fitting of the crystal structure of an H7 HA1 trimer (PDB ID: 4LN3, red, cartoon representation) into the cryoEM density map of a transparent HA1 trimer region. (e) Structure of the 16-nm S60-HA1 PVNP in two color schemes, showing the $S_{60}$ nanoparticle core (orange) and the 60 surface-displayed HA1 antigens (cyan), forming $20 \mathrm{HAl}$ trimers. (f) An $\mathrm{S}_{60}$ nanoparticle model (orange) based on the crystal structure of the $T=1$ VLP of feline calicivirus (PDB ID: 4PB6), showing the 60 exposed C-termini (green) of the $\mathrm{S}$ domain in triangle patterns (yellow dashed triangle). The corresponding HAl trimers are shown in the $\mathrm{S}_{60}-\mathrm{HAl}$ images in (a), (b), (d), and (e).

\subsection{Structures of the tag-free S-HA1 PVNPs}

Only the 21-nm PVNP was recognized in the major populations of the tag-free PVNPs. Its 3D structure was solved through cryoEM method at $15-\AA$ resolution, revealing the same $T=1$ icosahedral symmetry (Fig. 5) like the His-tagged PVNPs. Similar organization consisting of the $S_{60}$ nanoparticle inner shell and 60 exterior, trimer-like HA1 antigens like the His-tagged PVNPs were witnessed by fitting of the $S_{60}$-nanoparticle and HA1 trimer crystal structures into corresponding regions of the cryoEM density maps, and by structural comparisons between the tag-free and the His-tagged PVNPs (Figs. 3 and 5). However, differences between the tag-free PVNPs and the tagged ones were also noted. For example, the five-fold axis protrusions of the 21-nm/tag free PVNP go less outward compared with those of the 21-nm/Histagged PVNP (comparing Fig. 3(b) with Fig. 5(b)). As a result, the grooves among the five-fold axis protrusions of the $21-\mathrm{nm} /$ Histagged PVNP appear to be deeper than those of the 21-nm/tagfree PVNPs (Figs. 3(g) and 5(e)). We also noted that the roots of the HA1 antigens of the 21-nm/tag-free PVNP appear to submerge into the $\mathrm{S}_{60}$-nanoparticle wall unlike those of the 21nm/His-tagged one (comparing Fig. 3(b) with Fig. 5(b)). (a)

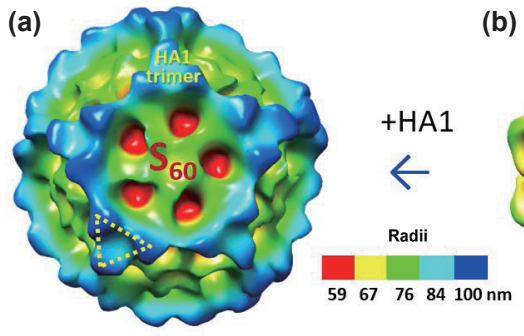

(b)

(c)

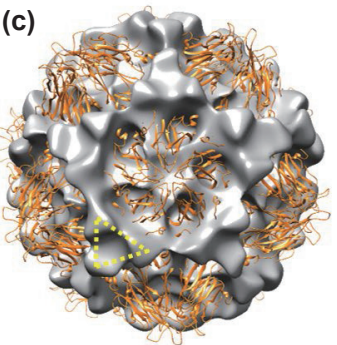

(d)
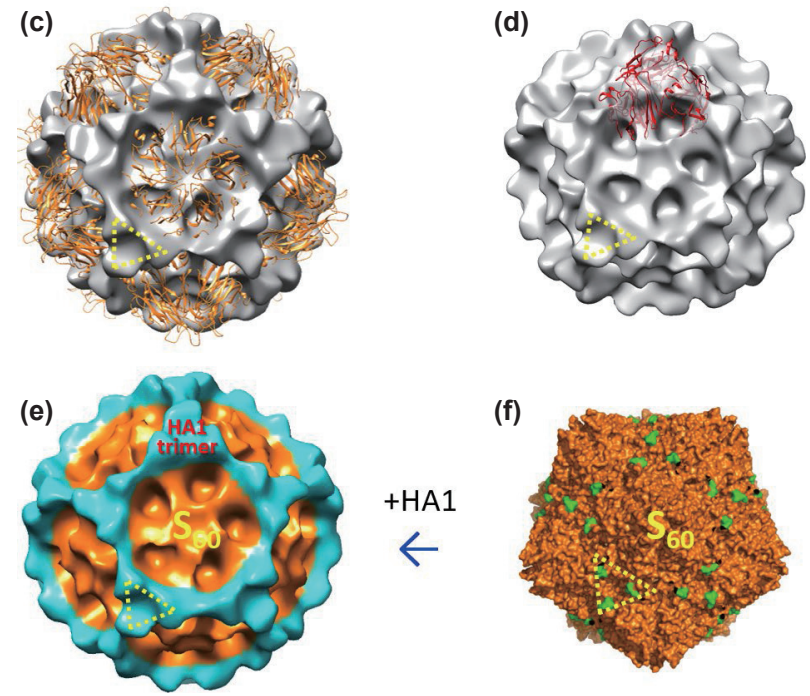

(f)

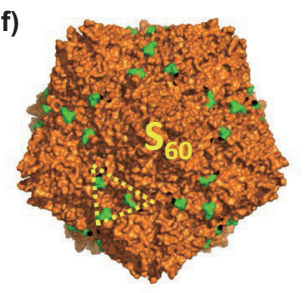

Figure 5 Structures of the tag-free, 21-nm S 60 -HA1 PVNPs. (a) and (b) The $3 \mathrm{D}$ structures of the 21-nm $\mathrm{S}_{60}-\mathrm{HA1}$ PVNP (a) and its $\mathrm{S}_{60}$ nanoparticle core region (b) reconstructed by cryoEM method, revealing a $T=1$ icosahedral symmetry. The images are shown at five-fold axes. The colored bar in between indicates the radii of the structures. A surface HAl trimer is indicated by a yellow dashed triangle. (c) Fitting of the crystal structure of the inner shell of the 60-valent feline calicivirus capsid (PDB ID: 4PB6, orange, cartoon representation) into the cryoEM density map of the $\mathrm{S}_{60}$ nanoparticle region. (d) Fitting of the crystal structure of the H7 HA1 trimer (PDB ID: 4LN3, red, cartoon representation) into the cryoEM density map of a surface HA1 trimer regions. (e) Structure of the $\mathrm{S}_{60}-\mathrm{HA1}$ PVNP in two color schemes, showing the $\mathrm{S}_{60}$ nanoparticle core (orange) and the 60 surface-displayed HAl antigens (cyan), forming $20 \mathrm{HAl}$ trimers. (f) $\mathrm{An}_{60}$ nanoparticle model (orange) based on the crystal structure of the $T=1$ VLP of feline calicivirus (PDB ID: 4PB6), showing the 60 exposed C-termini (green) of the $\mathrm{S}$ domain in triangle patterns (yellow dashed triangle). The corresponding HAl trimers are shown in the $\mathrm{S}_{60}-\mathrm{HAl}$ images in (a), (c), (d), and (e).

\subsection{Antigenic reactivity of the PVNPs to HA antibody}

EIA assays showed that the commercial antibody (Sino Biological) specific to IAV H7 HA recognized the His-tagged $S_{60}-\mathrm{HA} 1$ PVNPs, as shown by the high and dose-dependent EIA signals (Fig. 6(a)), indicating that the PVNP-displayed HA1s retained their natural conformations. These reactivity signals were mostly gone, when the PVNPs were boiled to destroy conformational epitopes, leaving mainly linear epitopes. As negative controls, the $\mathrm{S}_{60}$ nanoparticles without HA1 antigens did not react with the antibody (Fig. 6(a)). The tag-free PVNPs also revealed similar antigenic reactivity to the $\mathrm{H} 7 \mathrm{HA}$-specific antibody (data not shown). Due to the similarity of the two PVNP types, downstream experiments were performed using the His-tagged PVNPs by taking advantage of their easy purification with higher purity.

\subsection{Receptor binding function of the PVNP-displayed}

\section{HA1s}

The sialic acid binding ability and specificity of the $S_{60}$-HA1 PVNPs were determined by EIA-based glycan binding assays. The results showed that the PVNPs bound only 2,3-linked, but not 2,6or 2,8-linked sialoglycans (Fig. 6(b)), consistent with the known receptor binding nature of their parental H7N9 avian IVs [35, 37, 
(a) Reactivity to commercial $\mathrm{H} 7 \mathrm{HA}$ antibody

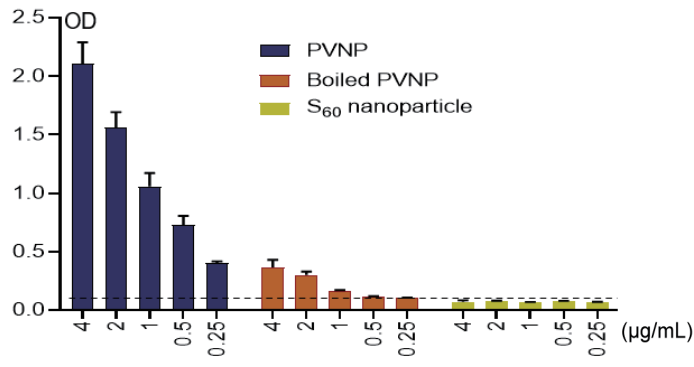

(b) Binding to specific sialoglycans

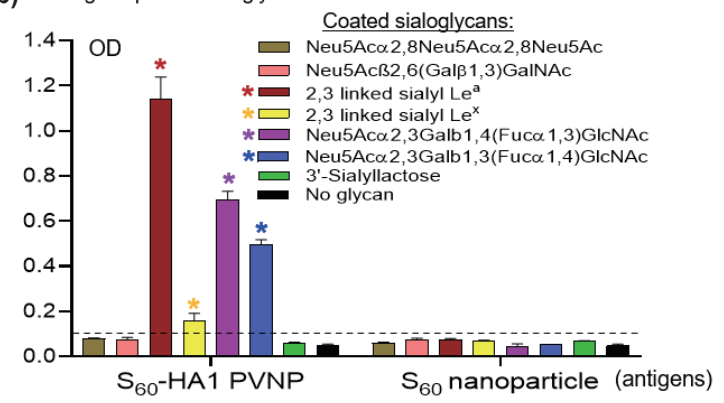

(c) Hemagglutination of human RBCs

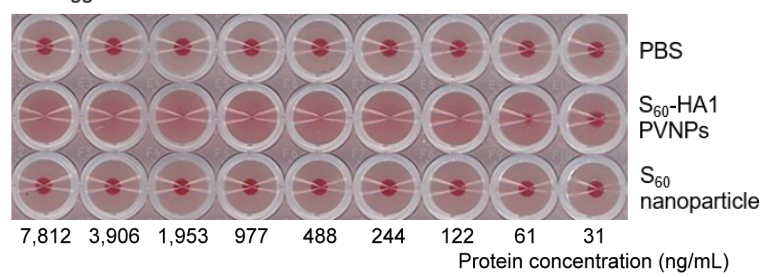

Figure 6 Antigenic reactivity and receptor binding function of the $\mathrm{S}_{60}$-HA1 PVNPs. (a) Antigenic reactivity of the $\mathrm{S}_{60}$-HA1 PVNPs to $\mathrm{H} 7 \mathrm{HA}$-specific antibody in EIA assays. $Y$-axis indicates EIA signals in optical density (OD), while $X$-axis shows the serially diluted PVNPs at indicated concentrations before (blue) and after (red) boiling treatment, using the $S_{60}$ nanoparticles (yellow) as controls. (b) Sialoglycans binding specificity of the $S_{60}$-HA1 PVNPs in EIAs. $Y$-axis indicates binding signals (OD), while $X$-axis shows various glycans with specific 2,3-, 2,6-, or 2,8-linked sialic acids. The positively binding glycans are indicated by star symbols. The $\mathrm{S}_{60}$ nanoparticle is used as negative controls. The cutoff signal value $(\mathrm{OD}=0.1$ ) is shown by a dashed line in both (a) and (b). (c) Hemagglutination of human erythrocytes by the $\mathrm{S}_{60}-\mathrm{HAl}$ PVNPs. The protein concentrations of the serially diluted $\mathrm{S}_{60}-\mathrm{HAl}$ PVNPs and the $S_{60}$ nanoparticles are shown at the bottom of the panel. The $S_{60}$ nanoparticles and $\mathrm{PBS}$ are used as negative controls.

38]. Accordingly, the $\mathrm{S}_{60}$-HA1 PVNPs agglutinated human RBCs at a high hemagglutination titer of $122 \mathrm{ng} / \mathrm{mL}$ (Fig. 6(c)). As negative controls, the $S_{60}$ nanoparticles did not bind any tested sialoglycans and did not cause hemagglutination. These data collectively demonstrated that the PVNP-displayed HA1 antigens retain the authentic structure, conformation, receptor binding function, and hemagglutination nature, supporting that the $\mathrm{S}_{60}{ }^{-}$ HA1 PVNPs may serve as a reagent for IV study.

\subsection{High immune response of the $S_{60}$-HA1 PVNPs}

Numerous literatures show that antigens with pathogen associated molecular patterns (PAMPs) in polyvalent nature induce stronger immune responses compared with the mono- or low-valent ones [39-42]. To prove that this is true for the PVNPs, mice were immunized with the $\mathrm{S}_{60}$-HA1 PVNPs using dimeric GST-HA1 proteins for comparison. After three immunizations, mouse sera were collected to determine HA1-specific IgG titers using recombinant $\mathrm{H} 7 \mathrm{HA} 1$ protein (Sino Biological) as capture antigen. The outcomes showed that the PVNP-elicited HA1-specific IgG titer $(80,000)$ was 8 -fold higher than that $(10,000)$ induced by the GST-HA1 protein $(P=0.0018$, Fig. $7(\mathrm{a}))$, supporting the above notion.
3.8 High hemagglutination inhibition (HI) effects of the PVNP immunized mouse sera

We then measured the inhibition effects of the mouse sera against hemagglutination of the $\mathrm{S}_{60}$-HA1 PVNPs, revealing high $\mathrm{HI}$ titers $(44,373)$ of the PVNP-immunized sera that was about 9.5 folds

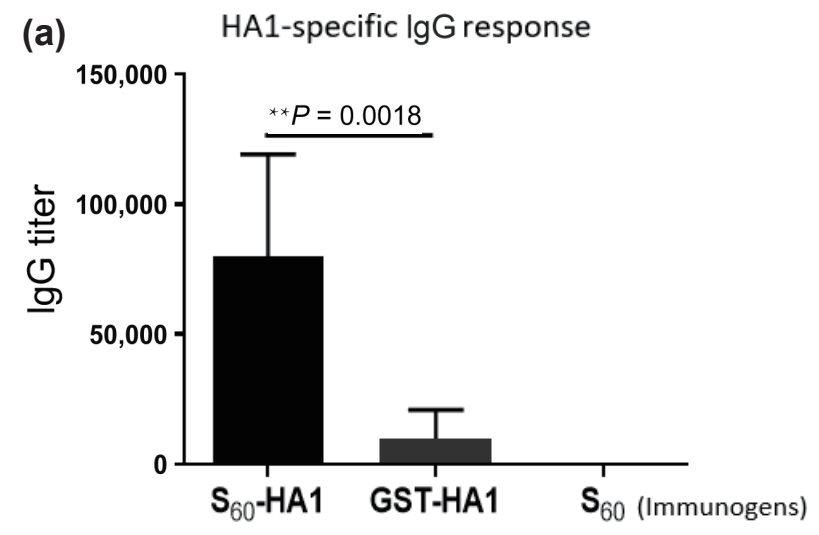

(b)

$\mathrm{HI}$ against PVNP hemagglutination

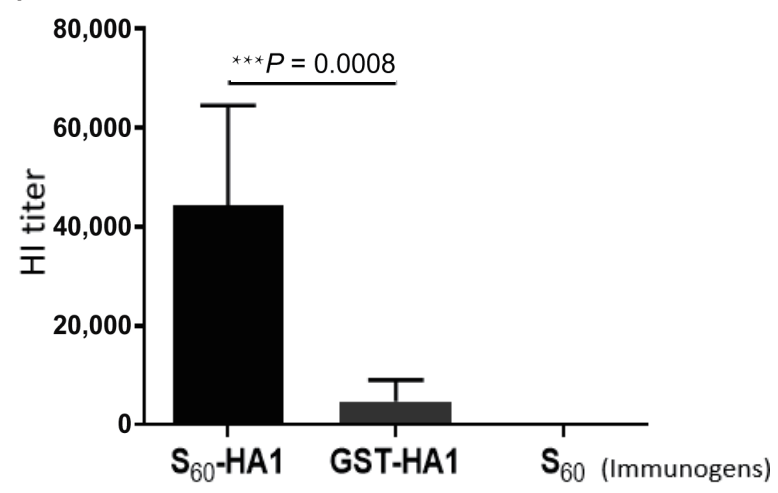

$\mathrm{HI}$ against $\mathrm{HA}$ hemagglutination

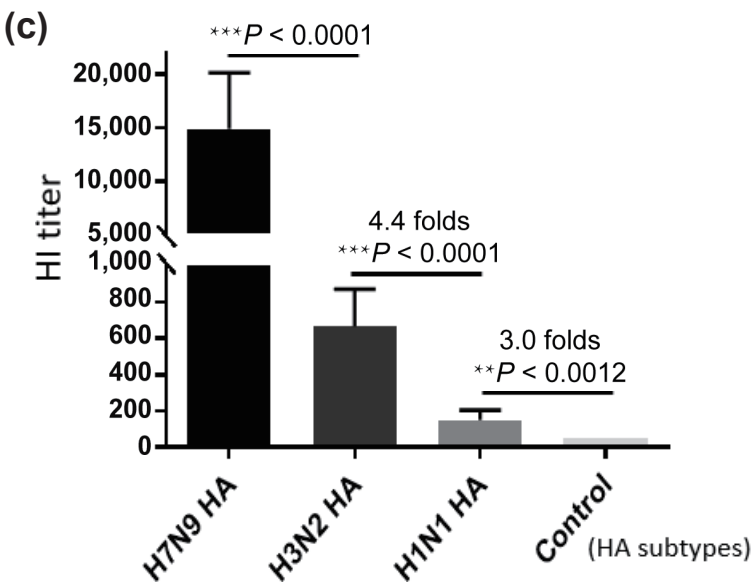

Figure 7 Immune responses of the $\mathrm{S}_{60}$-HA1 PVNPs in mice. (a) HA1-specific IgG titers elicited by the polyvalent $\mathrm{S}_{60}$-HA1 PVNPs $\left(\mathrm{S}_{60}-\mathrm{HA} 1\right)$ and the dimeric GST-HA1 fusion protein. (b) and (c) Hemagglutination inhibition (HI) titers of the PVNP- and GST-HA1-immunized mouse sera against hemagglutinations of the $\mathrm{S}_{60}-\mathrm{HAl}$ PVNPs (b) and commercial recombinant hemagglutinin (HA) proteins of H7N9, H3N2, and H1N1 subtypes (Sino Biological) (c). Y-axes indicate IgG titers (a) or $\mathrm{HI}$ titers (b) and (c), while $X$-axes indicate various immunogens (a), various immunogen-immunized mouse sera (b), or HA proteins of various subtypes used for the HI assays (c). The $S_{60}$ nanoparticles in (a) and the $\mathrm{S}_{60}$ nanoparticle-immunized mouse sera (b) (both labeled as $\mathrm{S}_{60}$ ) are negative controls. The control in (c) is the result of $\mathrm{HI}$ assay using the $\mathrm{S}_{60}$ nanoparticle-immunized mouse sera and the recombinant H7 HA protein. Statistic differences between data groups are shown as ${ }^{* *}$ for highly significant with $P$-values $<0.01$, and ${ }^{* *}$ for extremely significant with $P$-values $<0.001$. 
greater than that $(4,693)$ of the mouse sera after immunization with the GST-HA1 protein $(P=0.0008$, Fig. $7(\mathrm{~b}))$, consistent with their IgG titers. We also determined HI titers of the sera against hemagglutination of various commercial recombinant HA proteins (Sino Biological). The results showed that the $\mathrm{S}_{60}$-HA1 PVNP-immunized mouse sera showed very high HI titer $(14,933)$ against hemagglutination of the homologous H7 (A/ Zhejiang/1/2013/ H7N9) HA protein (Fig. 7(c)). Noteworthy, the sera also showed high $\mathrm{HI}$ titer (667) to heterologous subtype H3 (A/California/7/2004/H3N2) HA protein that clusters with the H7 subtype in the IAV group 2, but relatively low HI titer (150) to heterologous subtype H1 (A/NewYork/18/2009/H1N1) HA proteins that belongs to the IAV group 1 (Fig. 7(c)). As negative controls, the $\mathrm{S}_{60}$ nanoparticle did not elicit HA1-specific antibody (Fig. 7(a)) and the resulted mouse sera did not inhibit the hemagglutinations by the $\mathrm{S}_{60}$-HA1 H7 PVNPs (Fig. 7(c), control) or other tested recombinant HA proteins (data not shown). The above data support that the $\mathrm{S}_{60}-\mathrm{HA} 1$ PVNPs may serve as a flu vaccine candidate.

\section{Discussion}

In this study we designed and generated the $\mathrm{S}_{60}-\mathrm{HA1}$ PVNPs by taking advantage of the self-formation of our recently invented $S_{60}$ nanoparticles [20] to display the key neutralizing HA1 antigens of IVs for enhanced immunogenicity. The self-assembled $\mathrm{S}_{60}$-HA1 PVNPs can be produced through the prokaryotic E. coli system, as well as the eukaryotic mammalian cell system (to be published data). The bacterially expressed PVNPs have been well characterized structurally and functionally in this study. Each PVNP consists of an $\mathrm{S}_{60}$ nanoparticle core that resembles the inner shell of NoV capsid and 60 surface-displayed HA1 antigens extending from the inner shell, making the PVNPs resembling virus-like particles. Remarkably, the exposed HAl antigens are arranged into trimers, like those on the HAs of authentic flu viruses. Functional data showed that the PVNPs were recognized by HA-specific antibody, bound specific sialic acid receptors, and agglutinated human RBCs. These outcomes indicated that the PVNP displayed HA1 antigens retain the original structures, conformational epitopes, receptor binding functions, and PAMPs. Thus, the $\mathrm{S}_{60}$-HA1 PVNPs may be used as reagents to study IVs.

As a result of polyvalent presentations of the HA1 antigens by the PVNPs, and the well-preserved PAMPs of both HA1 antigens and $\mathrm{NoV} \mathrm{S}_{60}$-nanoparticles, the $\mathrm{S}_{60}$-HA1 PVNPs elicited strong immune responses. These were shown by the high HA1-specific IgG titers in the PVNP immunized mouse sera, as well as their high $\mathrm{HI}$ titers against hemagglutination of the $\mathrm{S}_{60}$-HA1 PVNPs and various recombinant $\mathrm{HA}$ proteins. The mouse sera after immunization with the $\mathrm{S}_{60}$-HA1 PVNPs exhibited very high $\mathrm{HI}$ titers, reaching to 14,933 , against hemagglutination of the homologous H7 HA proteins. This strongly suggested that the $\mathrm{S}_{60^{-}}$ HA1 PVNPs may serve as a potent vaccine candidate against $\mathrm{H} 7$ IAVs, such as the highly pathogenic H7N9 avian IAVs that caused five epidemic waves from 2013 to 2017 in China, claiming 511 human lives [43]. The high HI titer resulted apparently from the specific binding of the serum antibody to the HAl antigens of the H7 HA proteins and thus inhibited the hemagglutination ability of the HA protein. Same principle should also apply in vivo to confer protection. Due to the lack of an animal biosafety level 3 (BSL3) facility here, we are reaching out to identify collaborators with an appropriate animal model to study the protective efficacy of our $\mathrm{S}_{60}$-HA1 H7 PVNP vaccine candidate. If it succeeds, our $\mathrm{S}_{60}$-HA1 $\mathrm{H} 7$ PVNPs will serve as a bird flu vaccine to be used in poultry farms and/or high-risk human populations, such as those who have contact or work with poultry.
Noteworthy, the $\mathrm{S}_{60}$-HA1 PVNP-immunized mouse sera also inhibited hemagglutination of heterologous $\mathrm{H} 3$ (A/California/7/2004/H3N2) HA protein with a high HI titer of 667, which was significantly higher than that (150) against $\mathrm{H} 1$ (A/New York/18/2009/H1N1) HA proteins $(P<0.0001)$. These data implied certain cross protection of future $\mathrm{S}_{60}-\mathrm{HA} 1 \mathrm{H} 7 \mathrm{PVNP}$ vaccine against the two heterologous IAV subtypes. The higher HI titers of the H7 PVNP-immunized sera against the H3 HA protein than that of the H1 HA protein may be explained by the facts that $\mathrm{H} 7$ and $\mathrm{H} 3$ subtypes are genetically closer related, clustering in the same IAV group 2, while H1 belongs to IAV group 1, being genetically further away from the $\mathrm{H} 7$ subtype.

IV HA1 antigens are known for their variable sequences, leading to antigenic drifts and epochal evolution of flu viruses. However, a conserved antigenic supersite has been reported at or near the receptor binding site (RBS) that is targeted by broadly neutralizing antibodies [44-46]. Similarly, by analyzing the HA1 sequences of major H1 IVs circulated over the past century, a naturally protective epitope of limited variability near the RBS has been identified and proposed as a target of a universal flu vaccine [47]. Displaying the HA1 domain alone by the polyvalent $S_{60}$ nanoparticle may elicit substantially high proportion of antibody specific to the antigenic supersite and/or the conserved protective epitope. This may explain the observed high cross subtype HI titers. In fact, a previous study showed that bacterially expressed, oligomerized HA1s elicited cross-clade protective immunity in ferrets [48]. Thus, while a PVNP cocktail with multiple HA1 antigens of predominant IVs may offer broad protection (see above), an alternative approach would be to generate a pool of PVNPs that display a collection of native and/or artificially designed protective epitopes of limited variability [47] for broad protection against designated IVs. Finally, to the question that the PVNP containing only HA1s without other IV antigens might not elicit complete protective immunity as the primary vaccination, we wish to mention that our PVNPs will be a good choice of a boosting vaccine, because vast majority of human adults have been infected and/or immunized with one or more IVs and/or flu vaccines and thus have already memories to the conserved IV epitopes. In this case, the immunity against the variable HA1 antigens of currently circulated IVs may be the key to confer protection against the new IV strains.

We noted that the PVNPs formed by the S-HA1 proteins were heterologous in sizes. This was not unexpected, because in vitro expression of NoV VP1s often leads to VLP formation at heterologous sizes, including those in $T=1(\sim 31 \mathrm{~nm}), 3(\sim 46$ $\mathrm{nm})$, and $4(\sim 50 \mathrm{~nm})$ icosahedral symmetry, consisting of 60,180 , and $240 \mathrm{NoV}$ VP1s, respectively $[49,50]$. By focusing on the major populations of the His-tagged H7 PVNPs, we have reconstructed the 3D structures of two PVNPs with distinct diameters (21 nm vs. $16 \mathrm{~nm}$ ) by cryoEM method, revealing the same $T=1$ icosahedral symmetry, being composed of 60 copies of the S-HA1 fusion proteins. This size difference appears to be resulted from their different ways of homotypic interactions among the S-HAl fusion proteins during PVNP assembly. We speculated that the even larger PVNPs observed in this study may represent those in $T=3$ icosahedral symmetry. Further study is necessary to clarify this scenario.

Despite size variations, the PVNPs should share the basic organization comprising of the icosahedral inner shell formed by the NoV S domains and the surface displayed HA1 antigens. As explained above, the polyvalent PVNPs and their well-preserved PAMPs enhance the immunogenicity of the HAl antigens, leading to the high immune responses towards the HAl antigens. Thus, the size variations should not affect the potential of the PVNPs as an excellent IV immunogen. Such size heterogeneity of 
PVNPs may be reduced by using a mammalian cell expression system that is known to provide better folding environments for viral structural proteins. More importantly, the HA1 antigens are glycosylated, the mammalian cell system offers a better chance to reconstruct their original glycosylation and other post translational modifications, making the resulted PVNPs a better flu vaccine candidate. On the other hand, the above explained protective epitope of limited variability near the RBS has been shown to be peptide epitope without glycosylation [47]. This justifies the observed high HI effects of the PVNP immunized sera in this study, supporting our ultimate goal to develop the PVNPs into a useful flu vaccine.

The readily available PVNPs displaying the HA1 antigens of $\mathrm{H} 7$ subtype in this study and those that will be generated in future studies based on our established procedures will offer convenient and useful reagents to study flu viruses. These PVNPs can be produced either via the prokaryotic $E$. coli system quickly at a low cost or through a eukaryotic system (our unpublished data) potentially for better structural and functional integrity of the HA1 antigens. During this study, we observed that the PVNPs remained structurally intact after storage at -80 and $-20{ }^{\circ} \mathrm{C}$ for at least a year, as well as $4{ }^{\circ} \mathrm{C}$ for at least six months (MT, unpublished data). In addition, the PVNPs were shown to retain their structural integrity after lyophilization and rehydration treatments (MT, unpublished data), offering a further useful approach for PVNP preservation. The PVNPs with polyvalent $\mathrm{HAl}$ antigens will be able to better mimic the HA features of flu viruses, providing stronger antigenic reactivity, greater receptor binding avidity, and higher immune responses, than those of commercially available recombinant monomeric or trimeric HA proteins. Thus, our PVNPs will offer new and better tools to study flu viruses.

\section{Conclusions}

A technology has been developed to generate the self-assembled $\mathrm{S}_{60}-\mathrm{HA} 1$ PVNPs displaying the key neutralizing HA1 antigens of IVs as a promising tool for IV study and as a potential flu vaccine candidate. These have been supported by our extensive characterizations in the structures, functions, and immune responses of the PVNPs. Our study offers a new strategy to study and fight the deadly flu illness and provide a multifunctional platform for novel vaccine development against other infectious diseases.

\section{Acknowledgment}

The research described in this study was supported by the National Institute of Allergy and Infectious Diseases (NIAID, No. R56 AI148426-01A1 to M. T.), Cincinnati Children Hospital Medical Center (CCHMC, Innovation Funds 2018-2020, GAP Fund 2020-2021, and Research Innovation and Pilot Grant 2020-2021 to M. T.), and the Center for Clinical and Translational Science and Training (CCTST) of the University of Cincinnati College of Medicine (Pilot Collaborative Studies Grant 2018-2019 to M. T.) that was supported by the National Center for Advancing Translational Sciences of the National Institutes of Health (No. UL1TR001425). We thank the Purdue Cryo-EM Facility (http://cryoem.bio.purdue.edu) for help in the 3D structure reconstruction of the PVNPs.

\section{References}

[1] WHO. Seasonal influenza represents a year-round disease burden [Online]. https://www.who.int/news-room/feature-stories/detail/8things-to-know-about-pandemic-influenza.

[2] Fischer II, W. A.; Gong, M.; Bhagwanjee, S.; Sevransky, J. Global burden of influenza as a cause of cardiopulmonary morbidity and mortality. Glob Heart 2014, 9, 325-336.

[3] Iuliano, A. D.; Roguski, K. M.; Chang, H. H.; Muscatello, D. J.; Palekar, R.; Tempia, S.; Cohen, C.; Gran, J. M.; Schanzer, D.; Cowling, B. J. et al. Estimates of global seasonal influenzaassociated respiratory mortality: A modelling study. Lancet 2018, $391,1285-1300$.

[4] Centers for Disease Control and Prevention. Seasonal influenza vaccine effectiveness, 2005-2020 [Online]. https://www.cdc.gov/ flu/vaccines-work/effectiveness-studies.htm (accessed 2020).

[5] Wu, N. C.; Zost, S. J.; Thompson, A. J.; Oyen, D.; Nycholat, C. M.; McBride, R.; Paulson, J. C.; Hensley, S. E.; Wilson, I. A. A structural explanation for the low effectiveness of the seasonal influenza H3N2 vaccine. PLoS Pathog 2017, 13, e1006682.

[6] Skowronski, D. M.; Janjua, N. Z.; De Serres, G.; Sabaiduc, S.; Eshaghi, A.; Dickinson, J. A.; Fonseca, K.; Winter, A. L.; Gubbay, J. B.; Krajden, M. et al. Low 2012-13 influenza vaccine effectiveness associated with mutation in the egg-adapted $\mathrm{H} 3 \mathrm{~N} 2$ vaccine strain not antigenic drift in circulating viruses. PLoS One 2014, 9, e92153.

[7] Paules, C. I.; Sullivan, S. G.; Subbarao, K.; Fauci, A. S. Chasing seasonal influenza - the need for a universal influenza vaccine. New Engl. J. Med. 2018, 378, 7-9.

[8] Gouma, S.; Zost, S. J.; Parkhouse, K.; Branche, A.; Topham, D. J.; Cobey, S.; Hensley, S. E. Comparison of human H3N2 antibody responses elicited by egg-based, cell-based, and recombinant proteinbased influenza vaccines during the 2017-2018 season. Clin. Infect. Dis. 2020, 71, 1447-1453.

[9] Zost, S. J.; Parkhouse, K.; Gumina, M. E.; Kim, K.; Perez, S. D.; Wilson, P. C.; Treanor, J. J.; Sant, A. J.; Cobey, S.; Hensley, S. E. Contemporary H3N2 influenza viruses have a glycosylation site that alters binding of antibodies elicited by egg-adapted vaccine strains. Proc. Natl. Acad. Sci. USA 2017, 114, 12578-12583.

[10] Levine, M. Z.; Martin, E. T.; Petrie, J. G.; Lauring, A. S.; Holiday, C.; Jefferson, S.; Fitzsimmons, W. J.; Johnson, E.; Ferdinands, J. M.; Monto, A. S. Antibodies against egg- and cell-grown influenza A(H3N2) viruses in adults hospitalized during the 2017-2018 influenza season. J. Infect. Dis. 2019, 219, 1904-1912.

[11] Rolfes, M. A.; Flannery, B.; Chung, J. R.; O'Halloran, A.; Garg, S.; Belongia, E. A.; Gaglani, M.; Zimmerman, R. K.; Jackson, M. L.; Monto, A. S. et al. Effects of influenza vaccination in the united states during the 2017-2018 influenza season. Clin. Infect. Dis. 2019, 69, 1845-1853.

[12] Kanekiyo, M.; Wei, C. J.; Yassine, H. M.; McTamney, P. M.; Boyington, J. C.; Whittle, J. R. R.; Rao, S. S.; Kong, W. P.; Wang, L. S.; Nabel, G. J. Self-assembling influenza nanoparticle vaccines elicit broadly neutralizing $\mathrm{H} 1 \mathrm{~N} 1$ antibodies. Nature 2013, 499, 102-106.

[13] Yassine, H. M.; Boyington, J. C.; McTamney, P. M.; Wei, C. J.; Kanekiyo, M.; Kong, W. P.; Gallagher, J. R.; Wang, L. S.; Zhang, Y.; Joyce, M. G. et al. Hemagglutinin-stem nanoparticles generate heterosubtypic influenza protection. Nat. Med. 2015, 21, 1065-1070.

[14] Kanekiyo, M.; Joyce, M. G.; Gillespie, R. A.; Gallagher, J. R.; Andrews, S. F.; Yassine, H. M.; Wheatley, A. K.; Fisher, B. E.; Ambrozak, D. R.; Creanga, A. et al. Mosaic nanoparticle display of diverse influenza virus hemagglutinins elicits broad B cell responses. Nat. Immunol. 2019, 20, 362-372.

[15] Boyoglu-Barnum, S.; Ellis, D.; Gillespie, R. A.; Hutchinson, G. B.; Park, Y. J.; Moin, S. M.; Acton, O.; Ravichandran, R.; Murphy, M.; Pettie, D. et al. Elicitation of broadly protective immunity to influenza by multivalent hemagglutinin nanoparticle vaccines. bioRxiv 2020.05.30.125179, 2020. Available at: https://doi.org/10.1101/2020.05.30.125179.

[16] Bale, J. B.; Gonen, S.; Liu, Y. X.; Sheffler, W.; Ellis, D.; Thomas, C.; Cascio, D.; Yeates, T. O.; Gonen, T.; King, N. P. et al. Accurate design of megadalton-scale two-component icosahedral protein complexes. Science 2016, 353, 389-394.

[17] D'Aoust, M. A.; Couture, M. M. J.; Charland, N.; Trépanier, S.; Landry, N.; Ors, F.; Vézina, L. P. The production of hemagglutininbased virus-like particles in plants: A rapid, efficient and safe response to pandemic influenza. Plant Biotechnol. J. 2010, 8, 607-619.

[18] Smith, G.; Liu, Y.; Flyer, D.; Massare, M. J.; Zhou, B.; Patel, N.; 
Ellingsworth, L.; Lewis, M.; Cummings, J. F.; Glenn, G. Novel hemagglutinin nanoparticle influenza vaccine with Matrix- $\mathrm{M}^{\mathrm{TM}}$ adjuvant induces hemagglutination inhibition, neutralizing, and protective responses in ferrets against homologous and drifted A(H3N2) subtypes. Vaccine 2017, 35, 5366-5372.

[19] Portnoff, A. D.; Patel, N.; Massare, M. J.; Zhou, H. X.; Tian, J. H.; Zhou, B.; Shinde, V.; Glenn, G. M.; Smith, G. Influenza hemagglutinin nanoparticle vaccine elicits broadly neutralizing antibodies against structurally distinct domains of H3N2 HA. Vaccines 2020, 8, 99.

[20] Xia, M.; Huang, P. W.; Sun, C.; Han, L.; Vago, F. S.; Li, K. P.; Zhong, W. M.; Jiang, W.; Klassen, J. S.; Jiang, X. et al. Bioengineered norovirus $\mathrm{S}_{60}$ nanoparticles as a multifunctional vaccine platform. ACS Nano 2018, 12, 10665-10682.

[21] Xia, M.; Huang, P. W.; Jiang, X.; Tan, M. Immune response and protective efficacy of the S particle presented rotavirus VP8* vaccine in mice. Vaccine 2019, 37, 4103-4110.

[22] Tan, M.; Cui, L. B.; Huo, X.; Xia, M.; Shi, F. J.; Zeng, X. Y.; Huang, P. W.; Zhong, W. M.; Li, W. W.; Xu, K. et al. Saliva as a source of reagent to study human susceptibility to avian influenza H7N9 virus infection. Emerg. Microbes Infect. 2018, 7, 1-10.

[23] Tan, M.; Jiang, X. The P domain of norovirus capsid protein forms a subviral particle that binds to histo-blood group antigen receptors. $J$. Virol. 2005, 79, 14017-14030.

[24] Tan, M.; Hegde, R. S.; Jiang, X. The P domain of norovirus capsid protein forms dimer and binds to histo-blood group antigen receptors. J. Virol. 2004, 78, 6233-6242.

[25] Xia, M.; Huang, P. W.; Jiang, X.; Tan, M. A nanoparticle-based trivalent vaccine targeting the glycan binding VP8* domains of rotaviruses. Viruses 2021, 13, 72.

[26] Tan, M.; Huang, P. W.; Xia, M.; Fang, P. A.; Zhong, W. M.; McNeal, M.; Wei, C.; Jiang, W.; Jiang, X. Norovirus P particle, a novel platform for vaccine development and antibody production. J. Virol. 2011, 85, 753-764.

[27] Wang, L. Y.; Huang, P. W.; Fang, H.; Xia, M.; Zhong, W. M.; McNeal, M. M.; Jiang, X.; Tan, M. Polyvalent complexes for vaccine development. Biomaterials 2013, 34, 4480-4492.

[28] Xia, M.; Wei, C.; Wang, L. Y.; Cao, D. J.; Meng, X. J.; Jiang, X.; Tan, M. Development and evaluation of two subunit vaccine candidates containing antigens of hepatitis E virus, rotavirus, and astrovirus. Sci. Rep. 2016, 6, 25735.

[29] Xia, M.; Wei, C.; Wang, L. Y.; Cao, D. J.; Meng, X. J.; Jiang, X.; Tan, M. A trivalent vaccine candidate against hepatitis E virus, norovirus, and astrovirus. Vaccine 2016, 34, 905-913.

[30] Huang, P. W.; Farkas, T.; Zhong, W. M.; Tan, M.; Thornton, S.; Morrow, A. L.; Jiang, X. Norovirus and histo-blood group antigens: Demonstration of a wide spectrum of strain specificities and classification of two major binding groups among multiple binding patterns. J. Virol. 2005, 79, 6714-6722.

[31] Tan, M.; Fang, P. A.; Xia, M.; Chachiyo, T.; Jiang, W.; Jiang, X. Terminal modifications of norovirus $\mathrm{P}$ domain resulted in a new type of subviral particles, the small $\mathrm{P}$ particles. Virology 2011, 410, $345-352$.

[32] Tan, M.; Fang, P. A.; Chachiyo, T.; Xia, M.; Huang, P. W.; Fang, Z. Y.; Jiang, W.; Jiang, X. Noroviral P particle: Structure, function and applications in virus-host interaction. Virology 2008, 382, 115-123.

[33] Punjani, A.; Rubinstein, J. L.; Fleet, D. J.; Brubaker, M. A. cryoSPARC: Algorithms for rapid unsupervised cryo-EM structure determination. Nat. Methods 2017, 14, 290-296.

[34] Burmeister, W. P.; Buisson, M.; Estrozi, L. F.; Schoehn, G.; Billet, O.; Hannas, Z.; Sigoillot, C.; Poulet, H. Structure determination of feline calicivirus virus-like particles in the context of a pseudooctahedral arrangement. PLoS One 2015, 10, e0119289.

[35] Shi, Y.; Zhang, W.; Wang, F.; Qi, J. X.; Wu, Y.; Song, H.; Gao, F.; Bi, Y. H.; Zhang, Y. F.; Fan, Z. et al. Structures and receptor binding of hemagglutinins from human-infecting H7N9 influenza viruses. Science 2013, 342, 243-247.

[36] Yang, H.; Carney, P. J.; Chang, J. C.; Villanueva, J. M.; Stevens, J. Structural analysis of the hemagglutinin from the recent 2013 H7N9 influenza virus. J. Virol. 2013, 87, 12433-12446.

[37] Xu, R.; De Vries, R. P.; Zhu, X. Y.; Nycholat, C. M.; McBride, R.; $\mathrm{Yu}$, W. L.; Paulson, J. C.; Wilson, I. A. Preferential recognition of avian-like receptors in human influenza A H7N9 viruses. Science 2013, 342, 1230-1235.

[38] Tharakaraman, K.; Jayaraman, A.; Raman, R.; Viswanathan, K.; Stebbins, N. W.; Johnson, D.; Shriver, Z.; Sasisekharan, V.; Sasisekharan, R. Glycan receptor binding of the influenza A virus H7N9 hemagglutinin. Cell 2013, 153, 1486-1493.

[39] Tan, M.; Jiang, X. Norovirus capsid protein-derived nanoparticles and polymers as versatile platforms for antigen presentation and vaccine development. Pharmaceutics 2019, 11, 472.

[40] Tan, M.; Jiang, X. Recent advancements in combination subunit vaccine development. Hum. Vaccin. Immunother. 2017, 13, $180-185$.

[41] Khudyakov, Y.; Pumpens, P. Nanoparticles of norovirus. In Viral Nanotechnology. Khudyakov, Y.; Pumpens, P., Eds.; CRC Press: Boca Raton, 2015; pp 363-371.

[42] Tan, M.; Jiang, X. Subviral particle as vaccine and vaccine platform. Curr. Opin. Virol. 2014, 6, 24-33.

[43] Su, S.; Gu, M.; Liu, D.; Cui, J.; Gao, G. F.; Zhou, J. Y.; Liu, X. F. Epidemiology, evolution, and pathogenesis of H7N9 influenza viruses in five epidemic waves since 2013 in China. Trends Microbiol. 2017, 25, 713-728.

[44] Ekiert, D. C.; Kashyap, A. K.; Steel, J.; Rubrum, A.; Bhabha, G.; Khayat, R.; Lee, J. H.; Dillon, M. A.; O'Neil, R. E.; Faynboym, A. M. et al. Cross-neutralization of influenza A viruses mediated by a single antibody loop. Nature 2012, 489, 526-532.

[45] Whittle, J. R. R.; Zhang, R. J.; Khurana, S.; King, L. R.; Manischewitz, J.; Golding, H.; Dormitzer, P. R.; Haynes, B. F.; Walter, E. B.; Moody, M. A. et al. Broadly neutralizing human antibody that recognizes the receptor-binding pocket of influenza virus hemagglutinin. Proc. Natl. Acad. Sci. USA 2011, 108, 14216-14221.

[46] Lee, P. S.; Ohshima, N.; Stanfield, R. L.; Yu, W. L.; Iba, Y.; Okuno, Y.; Kurosawa, Y.; Wilson, I. A. Receptor mimicry by antibody F045092 facilitates universal binding to the $\mathrm{H} 3$ subtype of influenza virus. Nat. Commun. 2014, 5, 3614.

[47] Thompson, C. P.; Lourenço, J.; Walters, A. A.; Obolski, U.; Edmans, M.; Palmer, D. S.; Kooblall, K.; Carnell, G. W.; O'Connor, D.; Bowden, T. A. et al. A naturally protective epitope of limited variability as an influenza vaccine target. Nat. Commun. 2018, 9, 3859 .

[48] Khurana, S.; Verma, S.; Verma, N.; Crevar, C. J.; Carter, D. M.; Manischewitz, J.; King, L. R.; Ross, T. M.; Golding, H. Bacterial HA1 vaccine against pandemic H5N1 influenza virus: Evidence of oligomerization, hemagglutination, and cross-protective immunity in ferrets. J. Virol. 2011, 85, 1246-1256.

[49] Jung, J.; Grant, T.; Thomas, D. R.; Diehnelt, C. W.; Grigorieff, N.; Joshua-Tor, L. High-resolution cryo-EM structures of outbreak strain human norovirus shells reveal size variations. Proc. Natl. Acad. Sci. USA 2019, 116, 12828-12832.

[50] Devant, J. M.; Hansman, G. S. Structural heterogeneity of a human norovirus vaccine candidate. Virology 2021, 553, 23-34. 